\title{
Agenesis of the inferior vena cava: A rare cause of recurrent venous thromboembolism
}

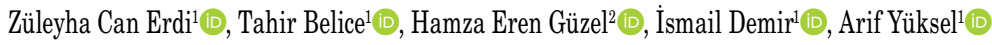 \\ ${ }^{1}$ Department of Internal Medicine, University of Health Sciences, Izmir Bozyaka Training and Research Hospital, Izmir, Turkey \\ ${ }^{2}$ Department of Radiology, University of Health Sciences, Izmir Bozyaka Training and Research Hospital, Izmir, Turkey \\ Received: August 10, 2020 Accepted: September 16, 2020 Published online: November 13, 2020
}

\section{ABSTRACT}

Agenesis of the inferior vena cava is a rare malformation. Inferior vena cava anomalies should be considered in the presence of recurrent thromboembolism or bilateral thromboembolism, particularly in young and middle-aged adults without any risk factors and family history. Herein, we present a case of agenesis of the inferior vena cava leading to recurrent venous thromboembolism in a middle-aged adult.

Keywords: Agenesis of inferior vena cava, thromboembolism, thrombosis.

Inferior vena cava (IVC) anomalies are extremely rare causes of deep vein thrombosis (DVT). The etiology of DVT must be specified, particularly in young patients. Young patients having bilateral DVT with no risk factor or family history are more likely to have IVC anomalies. ${ }^{[1]}$ Also, IVC anomalies may be asymptomatic and incidentally diagnosed with abdominal imaging methods. The diagnosis of IVC anomalies is important to prevent new thromboembolic events. In particular, in young patients, DVT should be taken into consideration as a rare complication of IVC.

Herein, we present a case of inferior vena cava agenesis (IVCA) leading to recurrent venous thromboembolism in a middle-aged adult patient.

\section{CASE REPORT}

A 47-year-old male patient was admitted to our outpatient clinic with abdominal pain and regurgitation. The patient had a history of recurrent bilateral lower extremity DVT since the age of 20 years and pulmonary embolism four years ago. He was on rivaroxaban treatment for eight months. Physical examination revealed that the patient had dilated varicose veins and edema in the bilateral lower extremities (Figure 1). There was no history of thrombosis in the family. In the laboratory examination, the following results were obtained:

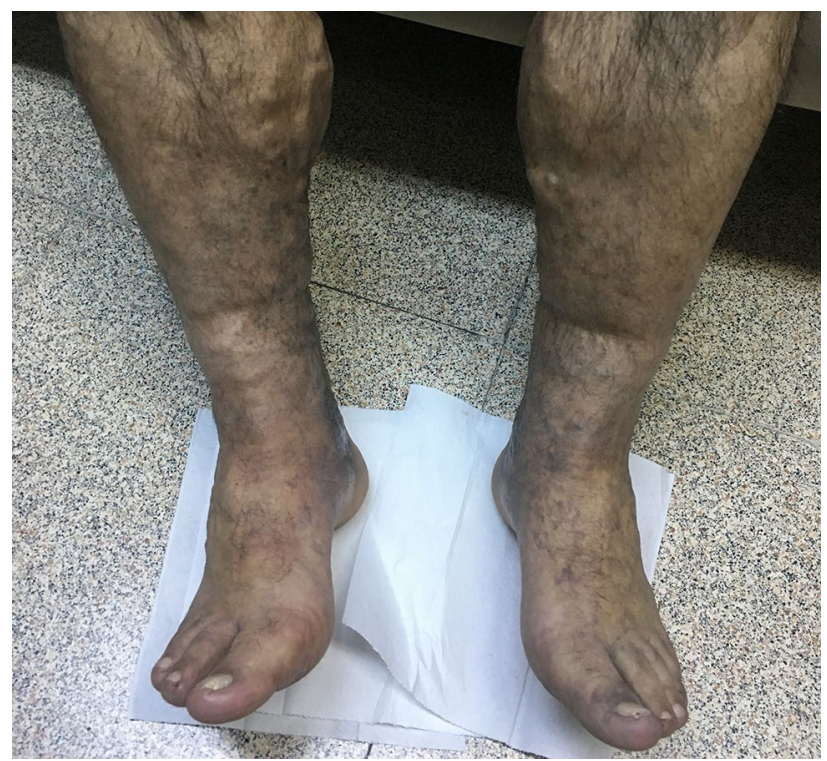

Figure 1. The patient had dilated varicose veins and edema in bilateral lower extremities.

Corresponding author: Züleyha Can Erdi, MD. SBÜ İzmir Bozyaka Ĕ̌itim ve Araştırma Hastanesi İç Hastalıkları Kliniği, 35170 Karabağlar, İzmir, Turkey.

Tel: +90 506 - 6055084 e-mail: zuleyhacan1@gmail.com

\section{Citation:}

Can Erdi Z, Belice T, Güzel HE, Demir İ, Yüksel A. Agenesis of the inferior vena cava: A rare cause of recurrent venous thromboembolism. Cardiovasc Surg Int 2020;7(3):182-185. 
white blood cells count $5.03 \times 10^{3}$ cells $/ \mu \mathrm{L}$; hemoglobin $13.8 \mathrm{~g} / \mathrm{dL}$; platelets $203 \times 10^{3} / \mathrm{L}$; C-reactive protein $1 \mathrm{mg} / \mathrm{L}$; amylase $106 \mathrm{U} / \mathrm{L}$, and lipase $118.1 \mathrm{U} / \mathrm{L}$. The thrombophilia blood panel was studied in the laboratory, and no pathology was found to cause any prothrombotic tendency (Factor II, Factor V Leiden, Factor XIII, PAI, MTHFR C677T and MTHFR A1298C). On previous Doppler ultrasonography of the lower extremity performed four months ago, there were wall irregularities secondary to lower extremity thrombus in the bilateral superficial and popliteal vein. A chronic thrombus was detected in a short segment of the tibial and popliteal vein. Abdominal ultrasonography, which was performed due to abdominal pain, revealed an adenoma of $20-\mathrm{mm}$ in diameter having a well-circumscribed margin on the right adrenal gland; and it was reported to be a suspicious lesion. Therefore, the patient underwent contrast-enhanced abdominal computed tomography (CT) scan, and numerous enlarged venous varicose veins were revealed in the paraaortic and paracaval space, presenting more prominently near the kidney on the left side (Figure 2a, b). Enlarged venous varicose structures
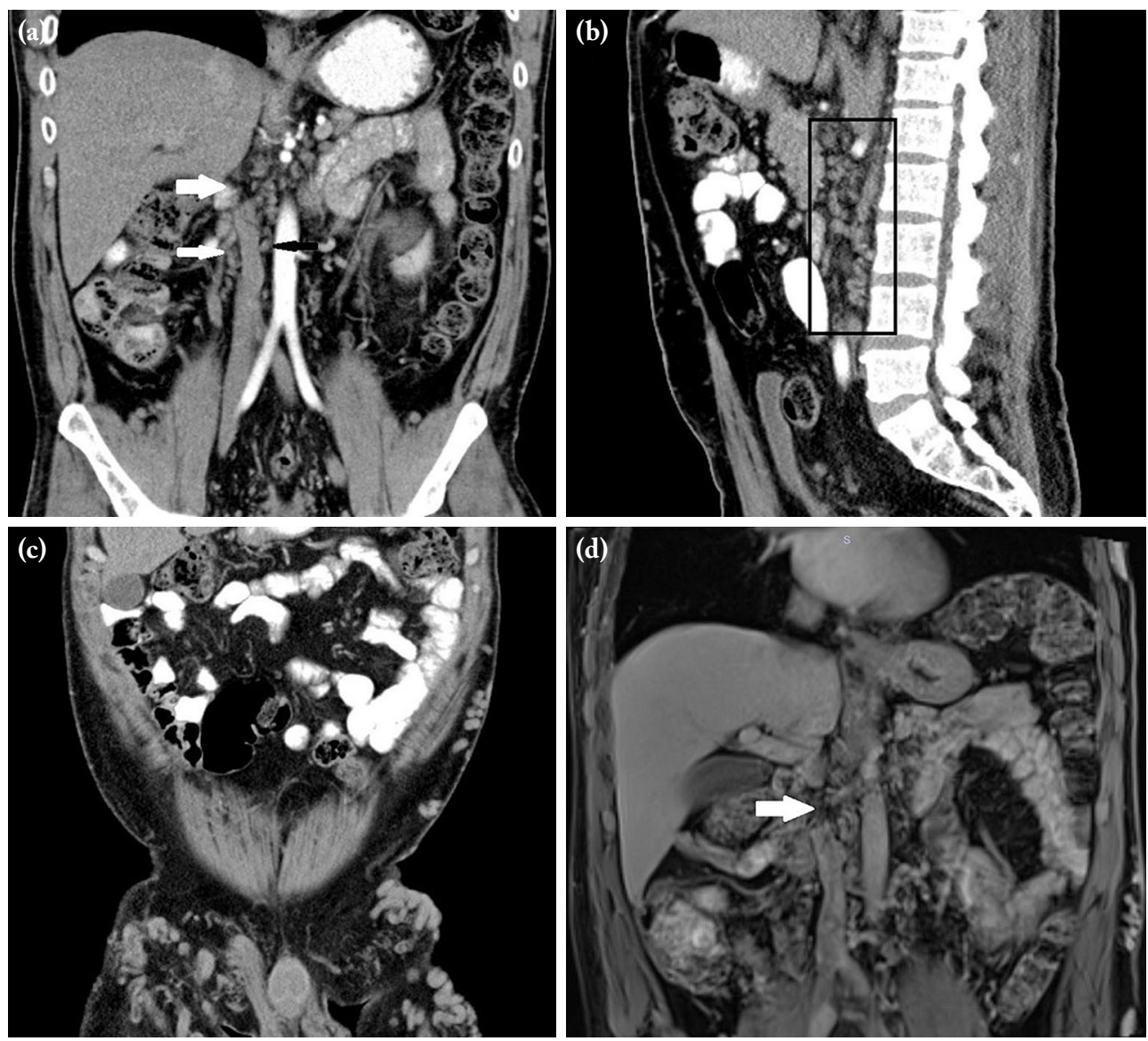

Figure 2. (a) In the coronal cross-sectional contrast-enhanced abdominal CT, the segment of inferior vena cava adjacent to lower contour of liver was not observed (thick arrow). On both sides of this segment, there were dilated and tortuous venous structures compatible with collateral vessels (thin arrows). (b) In the sagittal cross-sectional contrast-enhanced abdominal CT, prevertebral and retroperitoneal collateral dilated venous structures were observed. (c) In the coronal cross-sectional contrast-enhanced abdominal CT, numerous dilated and tortoise veins were observed under skin in both inguinal regions and anterior wall of abdomen on the left. (d) In the post-contrast late phase T1-weighted MR images, the segment of inferior vena cava adjacent to lower contour of liver was not observed.

CT: Computed tomography; MR: Magnetic resonance. 
were observed within the subcutaneous fat tissue of the anterior wall of the left abdomen and bilateral inguinal region (Figure 2c). The patient underwent abdominal magnetic resonance imaging and multiple venous collateralizations at the splenic hilum and left perirenal space, left renal hilum and left paraaortic and right paracaval region and segmental IVCA was observed (Figure 2d). The diameter of the IVC was found to be remarkably reduced. The relationship between the vena cava and left renal vein at the midline of the abdomen was consistent with the retrocaval renal vein, the changes related to IVCA, and collateral vessels. The diameter of the distal iliac veins was also found to be considerably reduced, particularly on the left side, and the left iliac vein was hypoplasic. It was evaluated as the segmental congenital IVCA. Thus, multidisciplinary evaluation was performed, and it was decided to monitor the patient with anticoagulant therapy without any surgical planning. A written informed consent was obtained from the patient.

\section{DISCUSSION}

The congenital IVCA is rare. It usually presents with idiopathic DVT of the lower extremity. ${ }^{[1,2]}$ In general population, the overall incidence of congenital IVCA is estimated to be 0.0005 to $1 \%$; however, it is found in 5\% of patients with idiopathic DVT aged $<30$ years. $^{[3]}$

The IVC develops between the sixth and eighth weeks of the embryonic life and contains three pairs of embryonic vein anastomosis, which are posterior cardinal veins, sub-cardinal veins, and supra-cardinal veins. ${ }^{[4]}$ A normal IVC transforms into a one-sided system on the right, comprising hepatic, prerenal, renal, and postrenal segments. ${ }^{[5]}$ As a result of the lack of this development during embryogenesis, IVC anomalies occur. Due to differences in this embryonic development, different types of IVC anomalies may occur. The IVC anomalies are seen nearly $0.5 \%$ of general population. ${ }^{[3]}$ Duplicated IVC and left IVC are the most common IVC anomalies. ${ }^{[6]}$ The IVCA is a rare variant and can be full or segmental. These anomalies may be associated with non-specific symptoms or completely asymptomatic, and the diagnosis is usually made incidentally during imaging studies. ${ }^{[7]}$

Insufficiency in collateral circulation in the IVC anomalies causes venous stasis and thromboembolic events. ${ }^{[5,8]}$ Although susceptibility to thromboembolism in IVCA is a well-defined entity, there is no gold standard treatment regimen. Oral anticoagulants are often used in the treatment. ${ }^{[9]}$

In appropriate cases, thrombolysis is combined.$^{[7]}$ Since IVC anomalies are associated with recurrent thromboembolism, patients should be informed that they should not remain immobile for long periods of time and they should refrain from oral contraceptive use. $^{[10]}$ Ultrasonography is often insufficient to detect IVC anomalies. Contrast-enhanced CT and magnetic resonance angiography should be used in the diagnosis. ${ }^{[11]}$

In conclusion, inferior vena cava anomalies should be considered in young and middle-aged patients with thromboembolism who do not have any risk factors or family history. As these patients may seek medical advice due to recurrent thromboembolic episodes, they can be treated with long-lasting anticoagulants. A close clinical follow-up is required for recurrent episodes and to observe the side effects of anticoagulant therapy. Therefore, it is of utmost importance to identify these patients using imaging studies to determine the etiology of the disease.

\section{Declaration of conflicting interests}

The authors declared no conflicts of interest with respect to the authorship and/or publication of this article.

\section{Funding}

The authors received no financial support for the research and/or authorship of this article.

\section{REFERENCES}

1. Ruggeri M, Tosetto A, Castaman G, Rodeghiero F. Congenital absence of the inferior vena cava: a rare risk factor for idiopathic deep-vein thrombosis. Lancet 2001;357:441.

2. Gayer G, Luboshitz J, Hertz M, Zissin R, Thaler M, Lubetsky A, et al. Congenital anomalies of the inferior vena cava revealed on CT in patients with deep vein thrombosis. AJR Am J Roentgenol 2003;180:729-32.

3. Parsa P, Lane JS 3rd, Barleben AR, Owens EL, Bandyk D. Congenital agenesis of inferior vena cava: a rare cause of unprovoked deep venous thrombosis. Ann Vasc Surg 2015;29:1017.e15-8.

4. Bass JE, Redwine MD, Kramer LA, Huynh PT, Harris JH Jr. Spectrum of congenital anomalies of the inferior vena cava: cross-sectional imaging findings. Radiographics 2000;20:639-52.

5. Obernosterer A, Aschauer M, Schnedl W, Lipp RW. Anomalies of the inferior vena cava in patients with iliac venous thrombosis. Ann Intern Med 2002;136:37-41.

6. Shin DS, Sandstrom CK, Ingraham CR, Monroe EJ, Johnson GE. The inferior vena cava: a pictorial review of embryology, 
anatomy, pathology, and interventions. Abdom Radiol (NY) 2019;44:2511-27.

7. Raymundo SRO, Cabral VS, Cavalieri RF, Reis Neto F. Thrombolysis for deep venous thrombosis associated with inferior vena cava agenesis in a young patient. BMJ Case Rep 2019;12:e229840.

8. Atmaca Usta H, Akbaş F, Erdenen F, Karagöz Y, Pekgüç E. Congenital agenesis of the inferior vena cava presenting with acute renal failure. Turk Gogus Kalp Dama 2014;22:182-6.
9. Arıkan AA, Emre S, Avni BF. The use of rivaroxaban in deep venous thrombosis associated with vena cava inferior agenesis. Turk Gogus Kalp Dama 2019;27:583-5.

10. Lambert M, Marboeuf P, Midulla M, Trillot N, Beregi JP, Mounier-Vehier C, et al. Inferior vena cava agenesis and deep vein thrombosis: 10 patients and review of the literature. Vasc Med 2010;15:451-9.

11. Aw-Zoretic J, Collins JD. Considerations for Imaging the Inferior Vena Cava (IVC) with/without IVC Filters. Semin Intervent Radiol 2016;33:109-21. 